\title{
Consideraciones en torno al enfoque de género en las Cooperativas No Agropecuarias en Pinar del Río
}

\author{
C. Orisel Hernández Aguilar \\ Doctora en Ciencias Jurídicas. Profesora Titular del Departamento de Derecho \\ de la Universidad de Pinar del Río, Cuba
}

Sumario: 1. A modo de introducción. 2. Acceso de las féminas a la condición de socias en las CNA de Pinar del Río. 3. Aporte de las socias a las CNA de Pinar del Río. 4. Participación de las socias en el retorno cooperativo en las CNA de Pinar del Río. 5. Acceso de las socias a cargos de dirección y estilos de dirección en las CNA de Pinar del Río. 6 . Autogestión de las condiciones y el régimen de trabajo y del descanso en las CNA de Pinar del Río. 7. Educación, capacitación e información en las CNA de Pinar del Río. 8. A modo de conclusiones. Bibliografía.

Resumen: El presente trabajo parte de la tesis de que los entes cooperativos, desde su naturaleza flexible, democrática y social pueden implementar, con mayor eficiencia, un mejor tratamiento de género. En Cuba, la introducción de las cooperativas no agropecuarias (CNA) ha generado importantes expectativas en tal sentido. Por tal razón, luego de cinco años de desarrollo de las CNA, conviene realizar un análisis para determinar cuánto se ha utilizado la potencia igualitaria de dichas figuras. Para ello, la exposición se ha centrado en el estudio de la realidad de Pinar del Río tratando, por separado, un conjunto de indicadores que permitirán comprender de forma general hasta dónde se ha llegado en la materialización del enfoque de género.

Palabras clave: cooperativas no agropecuarias, género y regulación jurídica.

Abstract: The present work is based on the thesis that cooperative entities, from their flexible, democratic and social nature, can implement, with greater efficiency, a better gender treatment. In Cuba, the introduction of non-agricultural cooperatives (CNA) has generated significant expectations in this regard. For this reason, after five years of development of the CNA, it is convenient to carry out an analysis to determine how much the equal power of said figures has been used. To this end, the exhibition focused on the study of the reality of Pinar del Río, dealing separately with a set of indicators that will allow a general understanding of the extent to which the materialization of the treatment of gender issues has materialized.

Keywords: non-agricultural cooperatives, gender and legal regulation. 


\section{A modo de introducción}

Los entes cooperativos, desde su naturaleza flexible, democrática y social pueden implementar, con mayor eficiencia, el tratamiento de género en su desarrollo, creando un beneficio - no solo empresarial- en su desenvolvimiento, sino reportando una importante contribución a la sociedad al propiciar un espacio adaptable a las necesidades de las féminas dentro del ámbito laboral, favoreciendo así su inserción y permanencia en estas organizaciones.

Dado el carácter relativamente reciente de la presencia de las cooperativas en los sectores no agropecuarios en Cuba, resultan insuficientes los estudios relativos a la relación que se genera entre las cooperativas no agropecuarias (CNA) y el fomento de condiciones laborales inclusivas y favorecedoras para las mujeres. Por ello, el presente trabajo centra la atención en analizar la experiencia de las CNA de la provincia de Pinar del Río en este particular.

Las reflexiones que se ofrecen atienden a elementos tales como aportes exigidos a las mujeres, acceso de estas a cargos de dirección, estilos de liderazgos asumidos, condiciones de trabajo, régimen de trabajo y descanso, participación en el retorno cooperativo ${ }^{1}$ y otros que ilustran en qué medida se han potenciado los aspectos que sirven, 0 pueden servir, para hacer de las CNA entidades ajustadas a las particularidades de este grupo social en el entorno nacional.

En la exposición que sigue se tratan por separado los indicadores seleccionados para procurar una determinación del nivel alcanzado en la materialización del tratamiento de las cuestiones de género dentro de las noveles cooperativas. Esta forma de construcción del discurso se ha seleccionado por razones puramente metodológicas, conociéndose la interrelación que existe entre los aspectos que se abordan, a fin de

1 Algunos de estos indicadores o criterios han sido asumidos como referentes en diversos estudios que han abordado la temática de enfoque de género. $V$. gr.: COCETA: Estudio sobre la realidad social y laboral de las mujeres en el cooperativismo de trabajo asociado en España, Madrid, 2004; Elıo Cemborain, E.: «Responsabilidad social en las cooperativas: igualdad de oportunidades entre hombres y mujeres», GEZKI, N. ${ }^{\circ} 2,2006$, pp. 35-71; Mateos de CABO, R., IturRioz del CAMPo, J. y Gimeno Nogués, R.: «La participación financiera y el papel de la mujer en la toma de decisiones de las sociedades cooperativas: los consejos de administración», Revista Europea de Dirección y Economía de la Empresa, vol. 18, núm. 3, 2009, pp. 65-82; Arroyo SánCHeZ, P.: «Perfil de la situación de la mujer en las cooperativas de trabajo en España», REVESCO n. ${ }^{\circ} 105,2011$, pp. 115142; COTULA, L.: Género y legislación. Los derechos de la mujer en la agricultura. FAO, Roma, 2007; y LASSALETTA GARCía, P.J.: El acceso a la condición de socio en la sociedad cooperativa de trabajo asociado. Junta de Andalucía, Madrid, 2010. 
sistematizar enfoques provenientes de diversas fuentes que aluden a los mismos.

De partida se asume la tesis de que, a pesar de las capacidades para potenciar un adecuado enfoque de género por parte de las cooperativas $^{2}$, no es esta su razón de ser. El objetivo prioritario de las cooperativas de trabajo asociado, en las cuales se centra la atención en este trabajo, «no ha sido conseguir una igualdad entre hombres y mujeres, sino entre el colectivo trabajador en general» ${ }^{3}$. Ello no implica que el movimiento cooperativo pretiriera las cuestiones de género, pero ciertamente en su dinámica se ha evidenciado una notable contradicción entre teoría y práctica, evidenciando la preponderancia de valores, principios y reglas masculinos ${ }^{4}$.

La preocupación por la igualdad entre los socios hombres y mujeres es una derivación, que podría considerarse natural, del principio de igualdad. De hecho, esto se consagra en la Ley Marco para las Cooperativas de América Latina (ACl-Américas, 2009) en su artículo 21, de lo que resulta una sensible limitante, al promover la «igualdad de género», que no es la aspiración idónea para una atención coherente de las múltiples connotaciones que tiene un término tan complejo como "género».

En este escenario general, y con sus características propias, se insertan las CNA cubanas, de las cuales las doce ${ }^{5}$ pinareñas hacen parte,

$2 \mathrm{Vid}$. HeRnÁndez Aguilar, O.: «La relación género y cooperativa en el ordenamiento jurídico cubano. Una aproximación a su realidad y a sus perspectivas ante el proceso de perfeccionamiento del modelo económico en el país». Boletín de la Asociación Internacional de Derecho Cooperativo, n. ${ }^{\circ} 46,2012$, pp. 297-320.

3 Elio Cemborain, E.: Perspectiva de género en el cooperativismo: retos y controversias, recuperado de http://www.euskonews.com/0641zbk/gaia64101es.html, en fecha 19 de junio de 2018.

4 Cfr. ApelQvist, K.: «Estrategias para una autoridad compartida entre hombres y mujeres en las cooperativas». Revista de Cooperación Internacional, n. ${ }^{\circ} 1,1996$, pp. 35-47.

5 Son ellas: la Cooperativa no Agropecuaria de Primer Grado Taller de Reparación de Calzado Estrella Roja (CNA-TRCER); la Cooperativa no Agropecuaria Casa Colonial (CNA-CC); la Cooperativa no Agropecuaria Restaurant Cielo Azul (CNA-RCA); la Cooperativa no Agropecuaria de Primer Grado Taller de Electrónica "Hermanos Cruz» (CNATEHC); la Cooperativa no Agropecuaria de Primer Grado Atelier «La Moda» (CNAALM); la Cooperativa no Agropecuaria de Primer Grado "Clavel Cooperativa» (CNA-C); la Cooperativa no Agropecuaria de Primer Grado Combinado Industrial Pinar del Río (CNA-CIPR); la Cooperativa no Agropecuaria de Primer Grado «Francisco Hernández Macurán» (CNA-FHM); la Cooperativa no Agropecuaria de Producción y Restauración de Bienes Muebles e Inmuebles (CNA-PRBMI); la Cooperativa no Agropecuaria de Primer Grado «Cooperativa Ornitológica de Cuba Pinar del Río» (CNA-COCPR); la Cooperativa no Agropecuaria de Primer Grado «Café Pinar» (CNA-CP); y la Cooperativa no Agropecuaria de Primer Grado Recuperación de Desechos Viñales (CNA-RDV). 
también con sus especificidades. En el acercamiento que se expone seguidamente, se analiza el marco legal vigente (en lo fundamental el Decreto-Ley n. ${ }^{\circ} 305$ «De las cooperativas no agropecuarias» (DL305/2012), y el Decreto n. ${ }^{\circ} 309$ «Reglamento de las cooperativas no agropecuarias de primer grado» (D309/2012)); los documentos propios de las CNA estudiadas, que figuran en el Registro de la Propiedad Mercantil de la Dirección Provincial del Ministerio de Justicia en Pinar del Río (RPM-DPJ-PR); artículos, ponencias, tesis y otros materiales elaborados por estudiosos de las materias en cuestión.

\section{Acceso de las féminas a la condición de socias en las CNA de Pinar del Río}

Las cooperativas, en tanto son organizaciones voluntarias, se conciben como entidades abiertas a todas las personas capaces de utilizar sus servicios y dispuestas a aceptar las responsabilidades de asociarse, sin discriminaciones de ninguna índole. Sin embargo, uno de los primeros aspectos que se constata con la lectura de la legislación vigente para las CNA, es que en ella prima un lenguaje sexista y excluyente, en el sentido de que se alude solo a los socios, excluyendo toda referencia a las socias ${ }^{6}$. Por ende, el acceso de las féminas se colige de la lógica general del ordenamiento jurídico cubano, que prohíbe la discriminación -incluida la que es motivada por razón del sexo de las personas ${ }^{7}$ - y cuenta con importantes regulaciones en defensa de los derechos de las mujeres, pero que aun adolece de una redacción que podría calificarse de tradicionalista ${ }^{8}$.

Partiendo de que ese manejo lingüístico no supone mayores inconvenientes prácticos, se asume que en el ingreso a las CNA prima la vo-

6 Cfr. Velázquez Quintián, S. y Barrera Rodríguez, S.: "Una mirada con enfoque de género a la legislación y la realidad de una cooperativa no agropecuaria cubana». Revista Idelcoop, n. ${ }^{\circ} 218,2016$, pp. 79-92.

7 Cfr. Capítulo VI «lgualdad», de la Constitución de la República de Cuba (vigente), Gaceta Oficial de la República de Cuba, Edición Especial, La Habana, 24/02/1976, reformada por la Ley de Reforma Constitucional, Gaceta Oficial de la República de Cuba, Edición Extraordinaria, n. ${ }^{\circ}$ 9, La Habana, 13/07/1992 y por la Ley de Reforma Constitucional, Gaceta Oficial de la República de Cuba, Edición Extraordinaria, n. 10, La Habana, 16/07/2002.

8 Vid. Silva González, J.L.: Fundamentos para una crítica propositiva al enfoque de género en el ordenamiento jurídico cubano: Estrategia para su implementación. Tesis presentada en opción al título de Licenciado en Derecho. Universidad de Pinar del Río, 2014. 
luntariedad (artículo 4, inciso a), DL305/2012), a pesar de reconocer que hubo limitaciones iniciales en el proceso de constitución de algunas de ellas ${ }^{9}$. Ello, unido al principio de puertas abiertas, implica que no haya obstáculos generales para la incorporación de las socias, salvo los que se desprenden de la propia naturaleza del principio y de puntuales incongruencias legales ${ }^{10}$.

Además, las condiciones objetivas del contexto cubano — gracias a las conquistas sociales alcanzadas luego de 1959- presenta como superadas algunas tradicionales desigualdades de género asociadas a la alfabetización, la formación por competencias, la propiedad de la tierra y el acceso al crédito, con lo cual no operan tales factores como limitaciones a la participación de las mujeres en las cooperativas.

No obstante, en la composición de la membrecía de las CNA analizadas hay un claro predominio masculino, siendo la excepción la cooperativa de mujeres CNA «Atelier La Moda» ${ }^{11}$ (CNA-ALM). Determinar las causas de dicha situación podría ser complicado, pues cada caso es único en sí mismo, pero debe considerarse con justeza en cada uno de ellos que la integración por sexos está fuertemente condicionada por la composición anterior de las entidades estatales de las que derivan ${ }^{12}$ y de la naturaleza de la actividad productiva que asumen como objeto social.

9 Vid. Piñeiro Harnecker, C.: Diagnóstico preliminar de las cooperativas no agropecuarias en La Habana, Cuba. Recuperado de https://www.ceec.uh.cu/file/569/ download?token=VfqEKMZE , el 18 de abril de2016 y Vuotro, M.: «Las cooperativas no agropecuarias y la transformación económica en cuba: políticas, procesos y estrategias». Revista de Estudios Cooperativos (REVESCO), recuperado de http://dx.doi.org/10.5209/ rev_REVE.2016.v120.49697, el 18 de abril de 2016.

10 Vid. Rodríguez Musa, O. y Hernández Aguilar, O.: «Apuntes críticos sobre la implementación jurídica del principio cooperativo de «adhesión voluntaria y abierta» en Cuba». Boletín de la Asociación Internacional de Derecho Cooperativo, No. 51, 2017, pp. 79-98.

11 Vid. Hernández Aguilar, O. y Valle Ríos, D.: «La Cooperativa No Agropecuaria Atelier «La Moda». Aproximación a la experiencia de una cooperativa de mujeres». Apuntes de Derecho Cooperativo para Cuba, Ediciones Loynaz, Pinar del Río, 2018, pp. 264 288.

12 Vid. Rodríguez Musa, O. y Rivera Gort, J.J. (2015): «Historia y realidad jurídica de la cooperativa en Cuba. Aproximación desde la experiencia práctica a las noveles cooperativas no agropecuarias en Pinar del Río». Boletín de la Asociación Internacional de Derecho Cooperativo, N. ${ }^{\circ} 49$, pp. 195-222. 
Tabla I

\section{Composición por sexos}

de los(as) asociados(as) a las CNA de Pinar del Río

\begin{tabular}{lcccccc}
\hline \multicolumn{1}{c}{ CNA } & $\begin{array}{c}\text { Total } \\
\text { inicial de } \\
\text { asociados }\end{array}$ & Socios & Socias & $\begin{array}{c}\text { Toral } \\
\text { actual de } \\
\text { asociados }\end{array}$ & Socios & Socias \\
\hline CNA-TRCER & 12 & 5 & 2 & 12 & 4 & 2 \\
CNA-CC & 12 & 7 & 5 & 10 & 4 & 6 \\
CNA-RCA & 9 & 4 & 5 & 9 & 4 & 5 \\
CNA-TEHC & 15 & 12 & 3 & 15 & 12 & 3 \\
CNA-ALM & 8 & - & 8 & 9 & - & 9 \\
CNA-C & 15 & 8 & 7 & 16 & 9 & 7 \\
CNA-CIPR & 19 & 17 & 2 & 18 & 16 & 2 \\
CNA-FHM & 17 & 14 & 3 & 24 & 20 & 4 \\
CNA-PRBMI & 10 & 8 & 2 & 11 & 9 & 2 \\
CNA-COCPR & 29 & 23 & 6 & 29 & 23 & 6 \\
CNA-CP & 12 & 10 & 2 & 13 & 10 & 3 \\
CNA-RDV & 7 & 7 & - & 6 & 6 & - \\
\hline
\end{tabular}

Fuente: Elaboración propia tomando como fuente los legajos del RPM-DPJ-PR.

\section{Aporte de las socias a las CNA de Pinar del Río}

El tercero de los principios cooperativos que sistematiza la Declaración sobre la Identidad Cooperativa de la $\mathrm{ACl}$ para delinear la institución, establece que: "Los asociados contribuyen equitativamente a la formación del capital de su cooperativa y lo administran democráticamente». De esta manera se orienta sobre la forma en que los/las socios(as) deben invertir en sus cooperativas y manejar sus resultados económicos.

En Cuba, el Decreto Ley 305/2012, exige que las CNA cuenten al momento de su constitución con un capital de trabajo inicial integrado a partir del aporte dinerario que realizan sus miembros y de los créditos bancarios que se otorguen con ese objetivo (artículo 21, DL305/2012). Además, se deja sentado que los/las socios(as), con independencia de cualquier otro aporte que hagan, están en la obligación de participar con su trabajo (artículo 23, DL305/2012).

Al respecto, se han suscitado importantes cuestionamientos sobre la admisibilidad de "otras aportaciones diferentes al dinero, dí- 
gase bienes, otros derechos distintos a los de créditos» ${ }^{13}$, a los cuales parece que no se cierra por completo el citado artículo 23. Entre los asuntos relativos a la materia por precisar figuran: "¿además del dinero y los derechos de créditos, qué más pudiera ser objeto de aportación? ¿En qué momento se pueden realizar las mismas? ¿Cómo se realizaría el proceso de valuación de esos aportes no dinerarios? ¿En qué concepto se realiza la aportación $?^{14}{ }^{15}$. La consecuencia práctica de esta regulación, puede constatarse —al menos en Pinar del Ríoen que los socios y socias de las CNA restrinjan sus aportes a dinero y trabajo.

De forma general no se hace distinción en cuando al sexo a la hora de disponer el monto a abonar, aunque al momento de su constitución, en dos de estas organizaciones, se admitieron - tanto a hombres como a mujeres- contribuciones de diferente cuantía. Esto supone, que por parte de los fundadores, se hicieron valoraciones sobre las particulares posibilidades de desembolso de efectivo de algunos de ellos, pudiendo incluirse en ellas razones vinculadas al género.

En el caso de las disposiciones estatutarias que fijan los montos mínimos para futuras incorporaciones, resultaría factible que se contemplaran reglas - de preferencia en un Reglamento interno- que ordenen efectuar reflexiones particularizadas ante cada nuevo ingreso, de manera que el tratamiento de género se enfocara como uno de los elemento a tener en cuenta.

13 Mesa TeJedA, N.T.: «Reflexiones críticas en torno a la regulación de las cooperativas no agropecuarias en Cuba». Boletín de la Asociación Internacional de Derecho Cooperativo, n. $.^{\circ} 48,2014$, pp. 227-243.

14 En este sentido puede resultar ilustrativa la Indicación Metodológica n. ${ }^{\circ} 4$. Dirección de Notarias del MINJUS de 3 de septiembre de 2015 que dispone se dé el nomen iuris de «Escritura pública de aporte al patrimonio de la Cooperativa no Agropecuaria de primer grado denominada...» al documento notarial que formalice el aporte a las cooperativas de bienes muebles o inmuebles sujetos a regulaciones especiales.

15 Mesa TeJedA, N.T.: ob. cit., p. 237. 
Tabla II

\section{Aportes de los/las asociados(as) a las CNA de Pinar del Río}

\begin{tabular}{|c|c|c|c|c|}
\hline CNA & $\begin{array}{l}\text { Aporte al momento } \\
\text { de la constitución } \\
\text { (cada socio) }\end{array}$ & $\begin{array}{l}\text { Aporte al momento } \\
\text { de la constitución } \\
\text { (cada socia) }\end{array}$ & $\begin{array}{c}\text { Aporte al posterior a } \\
\text { la constitución } \\
\text { (cada nuevo socio) }\end{array}$ & $\begin{array}{c}\text { Aporte al posterior a } \\
\text { la constitución } \\
\text { (cada nueva socia) }\end{array}$ \\
\hline CNA-TRCER & 200 CUP & 200 CUP & $\begin{array}{l}300 \text { CUP } \\
\text { (como monto } \\
\text { mínimo) }\end{array}$ & $\begin{array}{l}300 \text { CUP } \\
\text { (como monto } \\
\text { mínimo) }\end{array}$ \\
\hline CNA-CC & 170 CUP & 170 CUP & $\begin{array}{l}200 \text { CUP } \\
\text { (como monto } \\
\text { mínimo) }\end{array}$ & $\begin{array}{l}200 \text { CUP } \\
\text { (como monto } \\
\text { mínimo) }\end{array}$ \\
\hline CNA-RCA & 100 CUP & 100 CUP & $\begin{array}{l}1000 \text { CUP } \\
\text { (como monto } \\
\text { mínimo) }\end{array}$ & $\begin{array}{l}1000 \text { CUP } \\
\text { (como monto } \\
\text { mínimo) }\end{array}$ \\
\hline CNA-TEHC & 100 CUP & $\begin{array}{c}100 \text { CUP } \\
\text { (salvo una que } \\
\text { aporta } 200 \text { CUP) }\end{array}$ & $\begin{array}{l}100 \text { CUP } \\
\text { (como monto } \\
\text { mínimo) }\end{array}$ & $\begin{array}{l}100 \text { CUP } \\
\text { (como monto } \\
\text { mínimo) }\end{array}$ \\
\hline CNA-ALM & - & 168.75 CUP & - & $\begin{array}{l}200 \text { CUP } \\
\text { (como monto } \\
\text { mínimo) }\end{array}$ \\
\hline CNA-C & $\begin{array}{l}\text { A partir de un } \\
\text { monto mínimo } \\
\text { de } 40 \text { CUP es } \\
\text { diferente para } \\
\text { cada socio } \\
\end{array}$ & $\begin{array}{l}\text { A partir de un } \\
\text { monto mínimo } \\
\text { de } 40 \text { CUP es } \\
\text { diferente para } \\
\text { cada socia } \\
\end{array}$ & $\begin{array}{l}500 \text { CUP } \\
\text { (como monto } \\
\text { mínimo) }\end{array}$ & $\begin{array}{l}500 \text { CUP } \\
\text { (como monto } \\
\text { mínimo) }\end{array}$ \\
\hline CNA-CIPR & 100 CUP & 100 CUP & $\begin{array}{l}100 \text { CUP } \\
\text { (como monto } \\
\text { mínimo) }\end{array}$ & $\begin{array}{l}100 \text { CUP } \\
\text { (como monto } \\
\text { mínimo) }\end{array}$ \\
\hline CNA-FHM & 100 CUP & 100 CUP & $\begin{array}{c}\text { Si provienen de } \\
\text { la entidad estatal } \\
\text { que les dio } \\
\text { origen } 100 \text { CUP, } \\
\text { si no } 500 \text { CUP }\end{array}$ & $\begin{array}{c}\text { Si provienen de } \\
\text { la entidad estatal } \\
\text { que les dio } \\
\text { origen } 100 \text { CUP, } \\
\text { si no } 500 \text { CUP }\end{array}$ \\
\hline CNA-PRBMI & 100 CUP & 100 CUP & $\begin{array}{l}100 \text { CUP } \\
\text { (como monto } \\
\text { mínimo) }\end{array}$ & $\begin{array}{l}100 \text { CUP } \\
\text { (como monto } \\
\text { mínimo) }\end{array}$ \\
\hline CNA-COCPR & 250 CUP & 250 CUP & 250 CUP & 250 CUP \\
\hline CNA-CP & 500 CUP & 500 CUP & $\begin{array}{l}500 \text { CUP } \\
\text { (como monto } \\
\text { mínimo) }\end{array}$ & $\begin{array}{l}500 \text { CUP } \\
\text { (como monto } \\
\text { mínimo) }\end{array}$ \\
\hline CNA-RDV & 500 CUP & - & $\begin{array}{l}500 \text { CUP } \\
\text { (como monto } \\
\text { mínimo) }\end{array}$ & - \\
\hline
\end{tabular}

Fuente: Elaboración propia tomando como fuente los legajos del RPM-DPJ-PR. 


\section{Participación de las socias en el retorno cooperativo en las CNA de Pinar del Río}

Como resultado de sus aportes los/las cooperativistas pueden recibir beneficios de forma directa o inmediata, a través de la participación o utilización del servicio concebido como objeto social de la asociación, o de forma indirecta o mediata, en un momento posterior mediante el «retorno cooperativo». Por razones éticas y, también muchas veces, por disposición legal, al menos una parte importante de los excedentes ${ }^{16}$ no puede ser distribuida entre los asociados a ningún título, ni acrecentarán sus aportaciones individuales; sino que conformarán el patrimonio común de la entidad, reflejo de lo que se ha logrado en colectivo y para disfrutarlo en este concepto.

La posibilidad de que haya un retorno cooperativo repartible entre los/las miembros de las CNA está subordinada a la existencia de un balance positivo en términos de liquidez en el ejercicio, luego de la deducción de determinados montos que se asignan a finalidades tales como fondos, reservas y otras que se regulan en las normas generales o internas (artículo 57, D309/2012).

Según el artículo 60 del Decreto 309 «Los anticipos de las utilidades a los socios serán en efectivo, bienes o servicios». Sin embargo, en el modelo propuesto a los notarios del país, en el Seminario para los Notarios sobre los Requisitos Formales para la Constitución de Cooperativas de Primer Grado, de la Dirección de Notarías y Registros Civiles del Ministerio de Justicia, se consigna que «Los anticipos de las utilidades a los socios será en efectivo (además, según determinen pudiera ser en bienes o servicios)», dándole clara preferencia - con una interpretación restrictiva de la norma - al efectivo sobre las otras formas de compensación. Ello terminó, en el caso de la provincia de Pinar del Río, por traducirse en la reducción absoluta en este sentido que se constata en todos los estatutos adoptados por las CNA.

Otra confusión que no es atribuible en exclusiva a los cooperativistas es la relativa a la identidad entre salario y anticipo. En las indicaciones del Seminario citado cuando se propone el artículo relativo a la

16 Estos se definen como «sobrante del producto de las operaciones totales de la cooperativa, deducidos los costos y los gastos generales, las depreciaciones y provisiones, después de deducir ciertos porcentajes para fondos especiales del producto de las operaciones totales». BAstidas Delgado, O.: La Especificidad Cooperativa Apuntes para un Cooperativismo Alternativo, Centro de Estudios de la Participación, la Autogestión y el Cooperativismo (CEPAC), Universidad Central de Venezuela, 2009, p. 4. 
forma de cálculo del anticipo, si bien se reproduce la fórmula del Reglamento General, acto seguido se yerra al señalar que "Se calcula el anticipo mínimo y a partir de la estructura de la escala salarial vigente entre las diferentes categorías se le aplicaría esa estructura ${ }^{17}$ ». Aunque el documento plantea que se trata solo de una idea a considerar al no proponer ninguna otra en un contexto de limitado conocimiento del fenómeno cooperativo dio paso a una copia acrítica de ese modelo. De hecho, siete (CNA-ALM, CNA-TEHC, CNA-RCA, CNA-C, CNA-TRCER, CNA-CC y CNA-CP) de las 12 CNA de Pinar del Río en sus estatutos originales dispusieron suma fijas a devengar por cada socio(a) al mes en concepto de anticipo. Las cinco restantes no se excluyen porque lograran planificar reales anticipos, sino porque el nivel de oscuridad de los enunciados normativos no permite conocer con claridad el criterio asumido.

Estas insuficiencias son una consecuencia de un proceso que rebasa los límites territoriales de la provincia, y que está signado por la escaza conciencia cooperativa y la ponderación en la conciencia social del enfoque lucrativo, que conspiran en muchos casos contra una previsión equitativa de los términos del retorno ${ }^{18}$.

A medida que se ha ganado en conocimientos al respecto, se han realizado modificaciones en los estatutos de las referidas CNA. De hecho, seis de las doce CNA existentes han cambiado sus estatutos en este punto. No obstante, solo en cuatro (CNA-ALM, CNA-C, CNA-TRCER y (NA-CP) de estos casos se adopta una modalidad de cálculo de la participación a través de por cientos en función de las utilidades disponibles y las características de la labor del miembro. Para las otras dos entidades (CNA-COCPR y CNA-CNA-CC) no es posible, desde la lectura de lo previsto, determinar con exactitud qué método en particular se propone.

A pesar de todas estas imperfecciones, el tratamiento de hombres y mujeres en cuanto a la participación en el retorno es absolutamente

17 La cursiva es de la fuente original.

18 «...en comprobaciones realizadas por la Contraloría General de la República, (...) se detectaron cooperativas que el que menos ganaba, ganaba 3000 pesos, y el presidente 37000 pesos, catorce veces más». MURILLO JORGE, M.: «Intervención del miembro del Buró Político del Comité Central del Partido Comunista de Cuba y Jefe de la Comisión de Implementación y Desarrollo, en el X Periodo Ordinario de Sesiones de la VIII Legislatura de la Asamblea Nacional del Poder Popular, en el Palacio de Convenciones». Periódico Granma. 21 de diciembre de 2017. Recuperado de http://www.granma.cu/ cuba/2017-12-22/los-problemas-que-hemos-enfrentado-en-la-actualizacion-son-mascomplejos-y-mas-profundos-que-loque-habiamos-pensado-22-12-2017-02-12-01, el 22 de enero de 2018. 
equitativo. Esto se puede atribuir a lo entronizado que se encuentra en el ámbito laboral estatal el principio de igual trabajo, igual salario para hombres y mujeres, algo que también se extrapola, en sentido positivo, al ámbito cooperativo. Si en este sentido puede apreciarse alguna limitación, estaría vinculada a la superior valoración que se hace de la labor de los directivos de la cooperativa con respecto al resto de los/las socios(as)-trabajadores, pues en esta dinámica las femeninas resultan desfavorecidas dada su mejor presencia en los cargos de dirección.

\section{Acceso de las socias a cargos de dirección y estilos de dirección en las CNA de Pinar del Río}

Aun cuando en las cooperativas debe primar una dinámica organizativa participativa e igualitaria, distintos trabajos muestran que la presencia femenina en los órganos de gobierno de estas entidades es inferior a la masculina ${ }^{19}$.

En Cuba, las CNA cuentan con una estructura orgánica, integrada por: Asamblea General, Junta Directiva, Órgano de Administración y Comisión de Control y Fiscalización (artículos 33-45, D309/2012). Toda vez que en el primero de estos hay presencia de la universalidad de los miembros, se debe centra la atención en la composición que han tenido los restantes órganos.

En las entidades estudiadas, en clara correspondencia con su mayoritaria integración por socios hombres, se constata que en los cargos de dirección priman los asociados del sexo masculino, a los cuales, además, se les atribuyen con preferencia las responsabilidades de mayor peso - Presidente y Administrador-, reproduciendo claramente los estereotipos existentes en la sociedad. Este estado de cosas no es atribuible en modo alguno a la regulación vigente al respecto - que da iguales posibilidades a unos y otras para su incorporación a tales responsabilidades-, por lo cual las causas últimas de esta situación debe indagarse en el terreno de la subjetividad individual y colectiva de los/ las involucrados(as).

19 Vid. Esteban Salvador, M.L.: «¿Son las cooperativas más favorables a la presencia de mujeres en los consejos que otras entidades?». Revista de Estudios Cooperativos (REVESCO), n. ${ }^{\circ} 110,2013$, pp. 96-128. 
Tabla III

\section{Composición por sexos de cargos de dirección de las CNA de Pinar del Río}

\begin{tabular}{|c|c|c|c|c|c|}
\hline CNA & Presidente(a) & $\begin{array}{l}\text { Sustituto(a) del } \\
\text { Presidente }\end{array}$ & Secretario(a) & Administrador(a) & $\begin{array}{l}\text { Comisión } \\
\text { de Control y } \\
\text { Fiscalización }\end{array}$ \\
\hline CNA-TRCER & Hombre & Mujer & Mujer & - & Hombre \\
\hline CNA-CC & Hombre & Hombre & Mujer & Hombre & Hombre \\
\hline CNA-RCA & Hombre & Hombre & Mujer & Mujer & Mujer \\
\hline CNA-TEHC & Hombre & Hombre & Mujer & - & Hombre \\
\hline CNA-ALM & Mujer & Mujer & Mujer & - & - \\
\hline CNA-C & Hombre & Hombre & Hombre & Mujer & Hombre \\
\hline CNA-CIPR & Hombre & Hombre & Hombre & - & Hombre \\
\hline CNA-FHM & Hombre & Hombre & Mujer & Hombre & Mujer \\
\hline CNA-PRBMI & Hombre & Hombre & Hombre & Hombre & Mujer \\
\hline CNA-COCPR & Hombre & Hombre & Hombre & Hombre & Hombre \\
\hline CNA-CP & Hombre & $\begin{array}{l}\text { Hombre (inicial) } \\
\text { Mujer (actual) }\end{array}$ & Mujer & Hombre & Hombres \\
\hline CNA-RDV & Hombre & Hombre & Hombre & Hombre & Hombre \\
\hline
\end{tabular}

Fuente: Elaboración propia tomando como fuente los legajos del RPM-DPJ-PR.

La realidad descrita supone la presencia de un escenario en el cual no es posible aprovechar los beneficios que aportaría el liderazgo femenino. Coincidiendo con varios(as) estudiosos(as) del tema ${ }^{20}$, se puede sostener que los estilos de dirección de las mujeres son diferentes de los de los hombres, fundamentalmente por cuanto la presencia

20 Se suscriben —en general- las ideas sostenidas en: Esteban SALVAdoR, M.L., GarGallo CAstel, A. y Pérez SANZ, F.J.: "¿Presentan las cooperativas contextos favorables para la igualdad de género?: Especial referencia a la provincia de Teruel»». CIRIEC-España, Revista de Economía Pública, Social y Cooperativa, N. ${ }^{\circ} 88,2016$, pp. 61-92; Gómez ÁlVAREZ, J. y SÁnCHEZ BARRIOS, M.: "La participación de la mujer en los consejos de administración de empresas del Ibex-35». Revista de Humanidades, n. ${ }^{\circ} 16,2009$, pp. 1130 5029; KRISHNAN, H. y PARK, D.: "A few good women -on top management teams». Journal of Business Research, N. ${ }^{\circ}$ 58, 2005, pp.1712-1720; y RiBAS, M.: «Mujeres y cooperativismo en la Comunidad Autónoma del País Vasco». Revista Vasca de Economía Social, n. ${ }^{\circ} 2,2006$, pp.153-175. 
estas en los equipos directivos refuerzan la cooperación y el trabajo en equipo, lo que se revierte positivamente en los resultados de la organización.

A la complejidad - per se- de la temática relativa a los estilos de dirección, se le suma - en el caso de las CNA - las carencias que presentan las normas dinamizadoras de la vida cooperativa. En el diagnóstico inicial de PIÑEIRO HARNECKER —ya referido- se aprecia que no se ha establecido un tiempo mínimo para realizar la convocatoria a las asambleas generales de modo que haya suficiente tiempo para que la mayor cantidad de socios/socias puedan asistir, sobre todo si se tomarán decisiones importantes (véase, artículo 34, D309/2012); no se definen las atribuciones de la Junta Directiva (véase, artículo 45, D309/2012); y no se prevé la participación de mediadores en conflictos internos en la cooperativa.

Todo lo anterior ha supuesto que se perciba al «Presidente como una figura suprema» — aun cuando las normas definen a la Asamblea General como el órgano superior de la cooperativa - a manera de una "extrapolación de los esquemas de dirección que existen en el sector estatal» ${ }^{21}$. Con ello, por ende, se amplifica el efecto derivado de la hegemonía masculina en tales roles.

En Pinar del Río, esta situación se refleja con algunas singularidades. En la "Guía de Estatutos»(GE) recibida en la Dirección Provincial de Justicia para orientar el proceso de constitución de las CNA en su día -y que fuera adoptada como patrón mayoritariamente por las noveles cooperativas-, se precisaba que «Las convocatorias para las reuniones de los órganos de dirección y administración contendrán el orden del día y se realizarán con 72 horas de antelación» (Vid.: estatutos de CNA-TRCER, CNA-CC, CNA-RCA, CNA-TEHC, CNA-ALM, CNA-C, CNA-PRBMI, CNA-CP y CNA-RDV), superándose así (en 9 de las 12 (NA) una carencia que, como se refirió, ha sido regla en las experiencias de otros territorios, asegurando que todos los/las miembros del colectivo cooperativo estén en condiciones de planificar su participación efectiva en la toma de decisiones.

Este mismo documento-modelo previó que se establecieran rendiciones de cuentas ante la Asamblea General para el Presidente, el Administrador y la Comisión de Control y Fiscalización, marcando claramente que cada cooperativa debía fijar los períodos o momentos del año natural en que debía concretarse. En los estatutos examinados se aprecia, sin embargo, un reconocimiento del deber de rendir cuenta de

21 Mesa Tejeda, N.T.: ob. cit., p. 238. 
todos (CNA-CC, CNA-RCA, CNA-C, CNA-FHM, CNA-COCPR, CNA-CP y CNA-RDV) o algunos de estos (CNA-TRCER, CNA-TEHC, CNA-ALM, CNA-CIPR, CNA-PRBMI), pero no se dispone la periodicidad o el momento del año para hacerlo. En cualquier caso, este mecanismo - democrático y democratizador - es un complemento importante para contrarrestar los excesos de atribuciones que las normas generales presentan en favor de determinados órganos y, por ende, es preciso potenciarlos. En este sentido, en lugar de recurrir a una modificación estatutaria —que tiene complejidades particulares en su tramitación ${ }^{22}$-, podría optarse por el establecimiento de regulaciones particularizadas mediante un Reglamento interno de la CNA.

A pesar de las limitaciones del marco normativo general, las dos medidas previamente comentadas evidencian una intención de la entidad que ofrece el asesoramiento legal a las $\mathrm{CNA}^{23}$ de afianzar, en lo posible, el rol de la Asamblea General, lo que desafortunadamente no es suficiente, toda vez que no fue recepcionado de modo uniforme en los estatutos que se adoptaron, ni en las modificaciones posteriores de los mismos.

Otro aspecto que merece un comentario es la ausencia de Administrador en cuatro de las CNA pinareñas ${ }^{24}$. Esta preterición es notabilísima toda vez que el DL305/2012 dedica dos preceptos (artículo 17.3 y artículo 18) a dicha figura, el D309/2012 se ocupara de ella en la Sección Cuarta del Capítulo IV y la GE la recoge en una sección completa en su Capítulo III. Ante esta situación, y dado que el Administrador se subordina al Presidente (artículo 43.2, D309/2012), solo resta pensar que está

22 Las complejidades que se mencionan están asociadas al trámite notarial y registral que supone su inscripción, lo cual implica, además, una erogación patrimonial para las CNA. Cfr.: Hernández Aguilar, O., Acosta García, M. y Ramos Venero, A.B.: "La actividad registral mercantil relativa a las CNA. Análisis de su desarrollo en Pinar del Río». II Taller Internacional de Derecho Cooperativo, Pinar del Río, 7, 8 y 9 de noviembre de 2018.

23 La Empresa Provincial de Servicios Legales (EPSEL) de Pinar del Río ha mantenido un estrecho vínculo con la Universidad Hermanos Saíz Montes de Oca, haciendo posible el desarrollo de diversas actividades de superación de sus profesionales en el ámbito del Derecho Cooperativo. En la actualidad es el cliente de un Proyecto de Investigación encaminado a la elaboración de una "Metodología para perfeccionar el proceso de asesoría jurídica de las Cooperativas No Agropecuarias en la provincia de Pinar del Río, desde su gestación hasta su disolución»

24 Vid. supra Tabla III. La única explicación que pudiera ser plausible como alternativa a la realidad expuesta, sería la de considerar que aquellas personas que se integran a la CNA como socios, y que en las escrituras de constitución se consignan con la ocupación de «administrador», continuaron ejerciendo de hecho tal función, aun cuando no se hiciera notar en el documento notarial la adopción de acuerdo de la asamblea general en tal sentido. 
operando una acumulación de responsabilidad en la persona del Presidente, lo cual resultaría contraproducente para la dinámica cooperativa, en la que este cargo ya venía diseñado con una relevancia excesiva.

Si consideramos los elementos previamente expuestos, se constata un escenario en el que existen limitaciones objetivas y subjetivas que dificultan la materialización de los estilos de dirección matizados por la presencia femenina, toda vez que ellas no acceden a los cargos de las CNA con igual frecuencia que los hombres, quedando en su mayoría solo el espacio de las Asamblea para dar voz a las asociadas.

\section{Autogestión de las condiciones y el régimen de trabajo y del descanso en las CNA de Pinar del Río}

En principio, dada la finalidad de las cooperativas de velar -en primer lugar- por la satisfacción de los intereses de los/las asociados(as) y la comunidad, se ha planteado que es consustancial a su existencia la mejora en las condiciones de trabajo de sus miembros. Como correlato de ello, y en consonancia con la inspiración marxista del sistema socioeconómico y político cubano, sería posible para los socios, incluidas las socias mujeres, alcanzar el control sobre su trabajo y generar en un mejor clima psicosocial ${ }^{25}$.

No obstante, no figura en las normas generales que ordenan a las CNA pronunciamiento alguno al respecto, aunque se reconoce que ellas se orientan a "la satisfacción del interés social y el de los socios» (artículo 2, inciso 1, DL305/2012). De lo dispuesto solo puede colegirse que corresponde al «fondo de operaciones, inversiones, actividades socioculturales» la responsabilidad de solventar las mejoras, al menos materiales, en las condiciones laborales (artículo 57, inciso 3, D309/2012).

Al respecto de este particular solo se puede referir lo que establecen los estatutos que, como regla ${ }^{26}$, disponen únicamente el porciento de las utilidades no distribuidas a los/las socios(as) que les corresponden al fondo de operaciones, al de inversiones, al actividades socioculturales y al de contingencia, de conjunto con «otros». Sobre las formas en que se hace el manejo de estos fondos no hay regulación clara, haciéndose no-

25 Vid. Rodríguez MusA, O.: «Socialismo, Cooperativismo y Derecho. Dialéctica necesaria para la actualización del modelo económico cubano». Boletín de la Asociación Internacional de Derecho Cooperativo, N. ${ }^{\circ} 46,2012$, pp. 79-105.

26 De ello solo se excluye la CNA-COCPR, de la cual no consta regulación alguna en el sentido aludido, y la CNA-RDV que no asigna ninguna cuantía al fondo de inversiones. 
toria la necesidad de un Reglamento interno que, en cada CNA, ordene y de transparencia a la gestión asociada a los mismos, toda vez que el ordenamiento general no impone restricciones en este sentido.

Tabla IV

Fondos -expresados en el por ciento de las utilidades no distribuidas a los/las socios(as) que les corresponden- de las CNA de Pinar del Río

\begin{tabular}{lcccc}
\hline \multicolumn{1}{c}{ CNA } & $\begin{array}{c}\text { Fondo de } \\
\text { operaciones }\end{array}$ & $\begin{array}{c}\text { Fondo de } \\
\text { inversiones }\end{array}$ & $\begin{array}{c}\text { Fondo de } \\
\text { actividades } \\
\text { socioculturales }\end{array}$ & $\begin{array}{c}\text { Fondo de } \\
\text { contingencia } \\
\text { y otros }\end{array}$ \\
\hline CNA-TRCER & $30 \%$ & $\begin{array}{c}20 \% \text { (inicial) } \\
40 \% \text { (actual) }\end{array}$ & $\begin{array}{c}25 \% \text { (inicial) } \\
20 \% \text { (actual) }\end{array}$ & $\begin{array}{c}25 \% \text { (inicial) } \\
10 \% \text { (actual) }\end{array}$ \\
\hline CNA-CC & $5 \%$ & $10 \%$ & $5 \%$ & $10 \%$ \\
CNA-RCA & $5 \%$ & $10 \%$ & $5 \%$ & $10 \%$ \\
CNA-TEHC & $30 \%$ & $20 \%$ & $25 \%$ & $25 \%$ \\
CNA-ALM & $30 \%$ & $20 \%$ & $25 \%$ & $25 \%$ \\
CNA-C & $50 \%$ & $20 \%$ & $10 \%$ & $20 \%$ \\
CNA-CIPR & $50 \%$ & $20 \%$ & $15 \%$ & $15 \%$ \\
CNA-FHM & $50 \%$ & $20 \%$ & $15 \%$ & $15 \%$ \\
CNA-PRBMI & $50 \%$ & $20 \%$ & $15 \%$ & $15 \%$ \\
CNA-COCPR & - & - & - & $10 \%$ \\
CNA-CP & $5 \%$ & $10 \%$ & $5 \%$ & $68 \%$ \\
CNA-RDV & $30 \%$ & - & $2 \%$ & - \\
\hline
\end{tabular}

Fuente: Elaboración propia tomando como fuente los legajos del RPM-DPJ-PR)

Dada la información que figura en el RM-DPJ-PR no es posible determinar el alcance general que se le está dando a la utilización de los fondos de operaciones e inversiones, ni si existen esos «otros» distintos de los que tienen clara denominación, ya que solo hay inscripción registral de contadas actas y acuerdos de las Asambleas Generales de las CNA ${ }^{27}$. A partir de la documentación consultada solo se encon-

27 La inscripción registral de las actas o de la totalidad de los acuerdos de la Asamblea no es obligatoria según el marco regulatorio vigente que ordena la actividad registral de las CNA. La Indicación Metodológica de la Dirección de los Registros de la Propiedad, Mercantil y Patrimonio, del Ministerio de Justicia de 10 de junio de 2013 dispuso que los "restantes acuerdos adoptados» (aquellos que no se refieren a modificaciones estatutarias, incorporación de un nuevo socio y/o disolución y liquidación de la entidad) «podrán ser autenticados mediante Actas de Protocolización, permitiendo su acceso al Registro Mercantil», con lo cual queda reservado a la decisión particular de los asociados si se interesa este trámite o no. 
tró evidencia de alguna actividad en tal sentido en la CNA-ALM que por Acuerdo 4 de fecha 9 de enero de 2017 decidió brindarles servicio de cafetería a las socias y en la CNA-FHM que, según el punto 6 del Acta 50 de 5 de enero de 2018, decide destinar un $5 \%$ de lo restante luego de la distribución de las reservas obligatorias para el pago de certificados médicos y ayuda familiar a los socios ${ }^{28}$.

El impacto de estos dos sencillos acuerdos puede parecer pequeño, pero en cualquier caso deben apreciarse en su contexto. La CNA-ALM es una cooperativa solo de mujeres en un municipio tabacalero, donde las ofertas de cafetería resultan apreciables si se considera el ahorro con respecto al consumo en otras entidades de ese tipo, las distancias que separan a las viviendas de las socias de la sede de la cooperativa - en caso de que se considere la posibilidad de alimentarse en el hogar- y/o el alivio que supone a las asociadas no tener que preparar sus propios alimentos cada día de forma previa a la jornada laboral. Por su parte, la CNA-FHM opta por asegurar las condiciones psicosociales de los/las miembros(as) al destinar recursos a la ayuda familiar, de manera que estos pueden contar con apoyo extra que les permita afrontar dificultades que, en muchas ocasiones, implican la interrupción del vínculo laboral, temporal o definitivamente. Esta medida puede resultar más valiosa para las féminas, que como parte del estereotipo que se perpetúa en el entorno rural en que se ubica esta CNA, son las que asumen los sacrificios asociados a los roles tradicionalmente atribuido a las féminas como, por ejemplo, el de cuidadoras.

Otro apartado, abierto a la autorregulación cooperativa con una sensible repercusión en las dinámicas laborares de socios y socias es la relativa al régimen de trabajo y descanso. Los estudios de caso de algunas CNA confirman que subsiste en el país la doble jornada debido a la sobrecarga de trabajo doméstico de las mujeres ${ }^{29}$. En este sentido incide el olvido de las alternativas que ofrece la libertad de adaptar los estatutos de las cooperativas a las necesidades de sus miembros, pasando por alto la flexibilidad laboral empresarial interna. La flexibilidad interna puede abrir nuevas posibilidades, entre las que figuran la discontinuidad en la jornada cronológicamente —lo que afecta el horario

28 Nótese en este caso que, al modificar la distribución de los recursos y crear - aparentemente- un nuevo fondo, se ha incumplido con el procedimiento establecido al omitirse las formalidades de rigor, pues esto significa una alteración de lo establecido en los estatutos. Por tal razón, considerando el acuerdo en vigor, pero carente de valor legal fuera de la CNA hasta tanto se complete el proceso establecido por ley, no se refleja en la Tabla IV.

29 Vid. Velázquez Quintián, S. y Barrera Rodríguez, S.: ob. cit., p. 88. 
de trabajo, admitiendo el trabajo a tiempo parcial, trabajo por turnos y las horas extras - y el trabajo desde el hogar, entre otras ${ }^{30}$.

Al menos en el contexto que nos ocupa, se ha ignorado casi absolutamente esta noción de flexibilidad, reproduciendo los esquemas de organización del trabajo propios de las empresas estatales - de un promedio de 8 horas laborables por día, para un promedio semanal de 44 horas-. En ninguna medida esto es imputable a las normas generales, que nada disponen al respecto del tiempo de trabajo y descanso de los cooperativistas; ni a la GE, que dejó esos espacios en blanco en el prototipo de artículo que diseñó al efecto.

Las únicas CNA que se han desmarcado de la tendencia general han sido la CNA-ALM y la CNA-C. Ambas entidades mantienen los promedios semanales de horas laborables, realizando solamente una reorganización, en el caso de la primera para dejar libre el sábado - medida que beneficia a las socias para disponer de ese día para los quehaceres domésticos- y en el de la segunda, para que sean funcionales con las actividades que se prestan a los clientes.

Esta tendencia pone de manifiesto la preterición de la libertad de organizar las actividades por parte de los/las cooperativistas, no solo en términos de tiempo, sino también de localización. A menos que la secuencia de encadenamiento de la producción lo exija, nada se opone a que las labores a cumplimentar se hagan desde el hogar u otra ubicación y/o en un horario distinto del formalmente dispuesto, siempre que el trabajo se realice con calidad y se entregue en tiempo. Con ello podrían mantener el vínculo aquellos(as) que, por cuestiones personales o de otra índole, deban "ausentarse del puesto de trabajo» y se incentivaría la productividad de quienes, por cumplir con lo establecido, realizan sus actividades dentro de un horario y condiciones dados acusando cansancio, enfermedad $u$ otros padecimientos por estarles negada otra alternativa.

Lo anterior entronca directamente con lo tocante a la jornada de trabajo irregular. La GE previó un modelo de artículo que permitiría a la Asamblea General acordarlo «por la naturaleza propia del trabajo, por la complejidad de este o por voluntad de los socios». Aunque esta disposición se reitera, tal cual, en la totalidad de los estatutos, no hay constancia en el RM-DPJ-PR de acuerdos sociales que hagan uso de las mismas.

Para la regulación de las vacaciones anuales pagadas, la GE adoptó una fórmula cuestionable, dejando la previsión de las mismas y su

30 Vid. YÁÑEZ, S. "Consideraciones sobre flexibilidad laboral planteadas desde una mirada de género». En ENSIGNIA, J. y YÁÑEZ, S.: Sindicalismo, género y flexibilización en el Mercosur y Chile. Inserción laboral femenina. Fundación Friedrich Ebert (Representación Chile) y Centro de Estudios de la Mujer (CEM), Santiago de Chile, 1999, pp. 97-110. 
aprobación a cargo del órgano administrativo de la cooperativa. Esto contrasta con lo dispuesto, en el mismo prototipo de artículo, para los días de conmemoración nacional, oficial y feriados, los cuales se disfrutan según lo acuerden los socios reunidos en Asamblea General. A ello ha de sumarse que no se estableció un mínimo de tiempo de duración de las vacaciones en el año, que pudo ser de un mes, tomando como punto de referencia el vigente Código de Trabajo (artículos 78 y 101), pues si en las CNA los/las socios(as)-trabajadores(as) son a la vez empleadores(as) y empleados(as), no se justifica que tengan menos privilegios que aquellos que solo tienen la segunda condición ${ }^{31}$. Esta sería otra cuestión en la que a lo interno cada cooperativa podría establecer unas reglas mínimas que ordenaran los límites de las decisiones a adoptar por los órganos facultados por ley, haciendo relevante —una vez más- el tópico del Reglamento.

Los estatutos de la mayoría de las CNA imitan la GE en ese apartado. La diferencia la marcan la CNA-ALM y la CNA-COCPR que ordenan la forma en que los socios podrán disfrutar de sus vacaciones y la CNA-TRCER que restringe a 15 días el tiempo de descanso anual por concepto de vacaciones sus socios en una modificación a sus estatutos iniciales.

Tabla V

Regulación del régimen de trabajo y descanso en las CNA de Pinar del Río

\begin{tabular}{|c|c|c|c|}
\hline CNA & $\begin{array}{l}\text { Régimen de trabajo/ } \\
\text { Horario laboral }\end{array}$ & Jornada irregular & Vacaciones \\
\hline \multirow{2}{*}{ 昏 } & \multirow{2}{*}{$\begin{array}{c}8 \text { horas laborables por } \\
\text { día. } \\
44 \text { horas semanales } \\
\text { promedio. }\end{array}$} & \multirow{2}{*}{$\begin{array}{l}\text { A acordar según la natu- } \\
\text { raleza propia del trabajo, } \\
\text { la complejidad de este o } \\
\text { la voluntad de los socios. }\end{array}$} & $\begin{array}{l}\text { Vacaciones anuales paga- } \\
\text { das según estas sean pre- } \\
\text { vistas y aprobadas por el } \\
\text { órgano administrativo. }\end{array}$ \\
\hline & & & $\begin{array}{l}\text { Vacaciones } 15 \text { días al año } \\
\text { (actual). }\end{array}$ \\
\hline
\end{tabular}

31 Al respecto se suscribe la idea del profesor GARCíA Müller cuando afirma que en las cooperativas «las condiciones de trabajo y beneficios sociales de los trabajadores no son menores a la legislación laboral». GARCía Müller, A.: «¿Cómo determinar la identidad de una cooperativa?». II Taller Internacional de Derecho Cooperativo, Pinar del Río, 7, 8 y 9 de noviembre de 2018. 


\begin{tabular}{|c|c|c|c|}
\hline CNA & $\begin{array}{l}\text { Régimen de trabajo/ } \\
\text { Horario laboral }\end{array}$ & Jornada irregular & Vacaciones \\
\hline$\underset{\cup}{\cup}$ & $\begin{array}{c}8 \text { horas laborables por } \\
\text { día. } \\
44 \text { horas semanales } \\
\text { promedio. }\end{array}$ & $\begin{array}{l}\text { A acordar según la natu- } \\
\text { raleza propia del trabajo, } \\
\text { la complejidad de este o } \\
\text { la voluntad de los socios. }\end{array}$ & $\begin{array}{l}\text { Vacaciones anuales paga- } \\
\text { das según estas sean pre- } \\
\text { vistas y aprobadas por el } \\
\text { órgano administrativo. }\end{array}$ \\
\hline 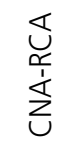 & $\begin{array}{c}8 \text { horas laborables por } \\
\text { día. } \\
44 \text { horas semanales } \\
\text { promedio. }\end{array}$ & $\begin{array}{l}\text { A acordar según la natu- } \\
\text { raleza propia del trabajo, } \\
\text { la complejidad de este o } \\
\text { la voluntad de los socios. }\end{array}$ & $\begin{array}{l}\text { Vacaciones anuales paga- } \\
\text { das según estas sean pre- } \\
\text { vistas y aprobadas por el } \\
\text { órgano administrativo. }\end{array}$ \\
\hline 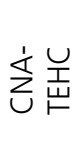 & $\begin{array}{c}8 \text { horas laborables por } \\
\text { día. } \\
44 \text { horas semanales } \\
\text { promedio. }\end{array}$ & $\begin{array}{l}\text { A acordar según la natu- } \\
\text { raleza propia del trabajo, } \\
\text { la complejidad de este o } \\
\text { la voluntad de los socios. }\end{array}$ & $\begin{array}{l}\text { Vacaciones anuales paga- } \\
\text { das según estas sean pre- } \\
\text { vistas y aprobadas por el } \\
\text { órgano administrativo. }\end{array}$ \\
\hline \multirow{2}{*}{ 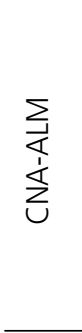 } & $\begin{array}{c}8 \text { horas laborables por } \\
\text { día. } \\
44 \text { horas semanales } \\
\text { promedio. }\end{array}$ & \multirow{2}{*}{$\begin{array}{l}\text { A acordar según la natu- } \\
\text { raleza propia del trabajo, } \\
\text { la complejidad de este o } \\
\text { la voluntad de los socios. }\end{array}$} & $\begin{array}{l}\text { Vacaciones anuales paga- } \\
\text { das según estas sean pre- } \\
\text { vistas y aprobadas por el } \\
\text { órgano administrativo. }\end{array}$ \\
\hline & $\begin{array}{c}9 \text { horas de lunes a } \\
\text { jueves. } \\
\text { y } 71 / 2 \text { el viernes } \\
\text { (actual) }\end{array}$ & & $\begin{array}{l}\text { Vacaciones cada } 51 / 2 \text { me- } \\
\text { ses o dos veces al año, en } \\
\text { periodos de } 12 \text { a } 24 \text { días } \\
\text { (actual). }\end{array}$ \\
\hline \multirow{2}{*}{$\sum_{\cup}^{\cup}$} & $\begin{array}{c}8 \text { horas laborables por } \\
\text { día. } \\
44 \text { horas semanales } \\
\text { promedio. }\end{array}$ & \multirow{2}{*}{$\begin{array}{l}\text { A acordar según la natu- } \\
\text { raleza propia del trabajo, } \\
\text { la complejidad de este o } \\
\text { la voluntad de los socios. }\end{array}$} & \multirow{2}{*}{$\begin{array}{l}\text { Vacaciones anuales paga- } \\
\text { das según estas sean pre- } \\
\text { vistas y aprobadas por el } \\
\text { órgano administrativo. }\end{array}$} \\
\hline & $\begin{array}{l}\text { Se ajustan las horas } \\
\text { según el área de } \\
\text { trabajo, manteniendo } \\
\text { los promedios iniciales } \\
\text { (actual). }\end{array}$ & & \\
\hline 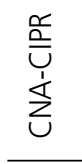 & $\begin{array}{c}8 \text { horas laborables por } \\
\text { día. } \\
44 \text { horas semanales } \\
\text { promedio. }\end{array}$ & $\begin{array}{l}\text { A acordar según la natu- } \\
\text { raleza propia del trabajo, } \\
\text { la complejidad de este o } \\
\text { la voluntad de los socios. }\end{array}$ & $\begin{array}{l}\text { Vacaciones anuales paga- } \\
\text { das según estas sean pre- } \\
\text { vistas y aprobadas por el } \\
\text { órgano administrativo. }\end{array}$ \\
\hline 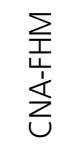 & $\begin{array}{c}8 \text { horas laborables por } \\
\text { día. } \\
44 \text { horas semanales } \\
\text { promedio. }\end{array}$ & $\begin{array}{l}\text { A acordar según la natu- } \\
\text { raleza propia del trabajo, } \\
\text { la complejidad de este o } \\
\text { la voluntad de los socios. }\end{array}$ & $\begin{array}{l}\text { Vacaciones anuales paga- } \\
\text { das según estas sean pre- } \\
\text { vistas y aprobadas por el } \\
\text { órgano administrativo. }\end{array}$ \\
\hline
\end{tabular}




\begin{tabular}{|c|c|c|c|}
\hline CNA & $\begin{array}{l}\text { Régimen de trabajo/ } \\
\text { Horario laboral }\end{array}$ & Jornada irregular & Vacaciones \\
\hline 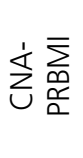 & $\begin{array}{c}8 \text { horas laborables por } \\
\text { día. } \\
44 \text { horas semanales } \\
\text { promedio. }\end{array}$ & $\begin{array}{l}\text { A acordar según la natu- } \\
\text { raleza propia del trabajo, } \\
\text { la complejidad de este o } \\
\text { la voluntad de los socios. }\end{array}$ & $\begin{array}{l}\text { Vacaciones anuales paga- } \\
\text { das según estas sean pre- } \\
\text { vistas y aprobadas por el } \\
\text { órgano administrativo. }\end{array}$ \\
\hline 安愛 & 一 & 一 & $\begin{array}{l}\text { Vacaciones de } 15 \text { días } \\
\text { primer semestre del año } \\
\text { y de } 15 \text { días segundo se- } \\
\text { mestre del año. }\end{array}$ \\
\hline 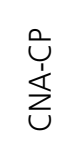 & $\begin{array}{c}8 \text { horas laborables por } \\
\text { día. } \\
44 \text { horas semanales } \\
\text { promedio. }\end{array}$ & $\begin{array}{l}\text { A acordar según la natu- } \\
\text { raleza propia del trabajo, } \\
\text { la complejidad de este o } \\
\text { la voluntad de los socios. }\end{array}$ & $\begin{array}{l}\text { Vacaciones anuales paga- } \\
\text { das según estas sean pre- } \\
\text { vistas y aprobadas por el } \\
\text { órgano administrativo. }\end{array}$ \\
\hline 京 & $\begin{array}{c}8 \text { horas laborables por } \\
\text { día. } \\
44 \text { horas semanales } \\
\text { promedio. }\end{array}$ & $\begin{array}{l}\text { A acordar según la natu- } \\
\text { raleza propia del trabajo, } \\
\text { la complejidad de este o } \\
\text { la voluntad de los socios. }\end{array}$ & $\begin{array}{l}\text { Vacaciones anuales paga- } \\
\text { das según estas sean pre- } \\
\text { vistas y aprobadas por el } \\
\text { órgano administrativo. }\end{array}$ \\
\hline
\end{tabular}

Fuente: Elaboración propia tomando como fuente los legajos del RPM-DPJ-PR.

Lo antes relacionado permite sostener que aún quedan muchas potencialidades por desarrollar en cuanto a la utilización coherente de la potestad de autorregulación de las cooperativas en relación a las condiciones y el régimen de trabajo y de descanso de los/las socios(as) para ser consecuentes con los fines cooperativos. La utilización de los fondos de operaciones, de inversiones, y los otros que se pueden crear, ha de pensarse de tal forma que se revierta en el aumento de la calidad del entorno socio-laboral de hombres y mujeres integrados a las CNA, toda vez que el centro de cualquier actuación cooperativa debe ser el mejor interés de sus miembros, entendidas también sus particulares necesidades -incluidas las de género-. En lo atiente a los regímenes de trabajo y descanso debe aprovecharse la flexibilidad interna que poseen estas entidades para favorecer a todos sus asociados(as) - particularmente los que asumen dobles jornadas y responsabilidades familiares de peso- , erigiéndose, efectivamente, en una forma superior de organización del trabajo. 


\section{Educación, capacitación e información en las CNA de Pinar del Río}

El principio de educación, capacitación e información cooperativa, según la $\mathrm{ACl}^{32}$, tiene múltiples destinatarios (miembros y sus familiares, los empleados o trabajadores, los dirigentes o directivos, el público), contenidos diferenciados (principios y doctrinas, las técnicas económicas, de gestión, profesionales, el entorno, etc.) y alcances variados (a lo interno y a lo externo de la cooperativa). Por ello, en perfecta síntesis, reza: "Las cooperativas brindan educación y capacitación a sus asociados, representantes elegidos, funcionarios y empleados, de manera que puedan contribuir efectivamente al desarrollo de ellas. Informan al público en general, particularmente a los jóvenes y a los líderes de opinión, acerca de la naturaleza y los beneficios de la cooperación».

Dada la significación de este principio, como regla de oro del cooperativismo, resulta llamativa — apunta PIÑEIRO HARNECKER - la ausencia en las disposiciones que ordenan a las CNA de un pronunciamiento expreso en tal sentido, lo cual se acompaña de la omisión de una comisión de educación y de un fondo particular para garantizar los gastos que toda labor educativa conlleva ${ }^{33}$.

No obstante, sería admisible cualquier actividad en este sentido a través de la interpretación del inciso f) del artículo 4 del DL305/2012, el cual preceptúa que las cooperativas se sustentan en el principio de responsabilidad social, contribución al desarrollo planificado de la economía y al bienestar de sus socios y familiares, y como parte de ellos «Trabajan por fomentar la cultura cooperativista y por las satisfacciones de las necesidades materiales, de capacitación, sociales, culturales, morales y espirituales de sus socios y familiares». Habría aquí que asumir en sentido amplio el alcance de los términos "cultura cooperativista» y «capacitación». En cuanto a la financiación de las acciones educativas, sería factible su sufrago con cargo al «fondo de de operaciones, inversiones, actividades socio-culturales», si no se hubiesen constituidos «otros» con tal finalidad por decisión adoptada en estatutos (artículo 57.3, D309/2012).

En el caso de Pinar del Río, como se evidencia en la Tabla IV, existen los fondos de actividades socio-culturales, pero no hay evidencias

32 Alianza Cooperativa Internacional. Declaración Sobre la Identidad Cooperativa, Manchester, 1995, recuperado de http://www.elhogarobrero1905.org.ar, en fecha 19 de diciembre de 2009.

33 Cfr. Piñeiro Harnecker, C.: ob. cit., p. 9. 
en los acuerdos inscritos en el RM-DPJ-PR de que se haga uso de estos en acciones de formación - de las cuales tampoco figura constancia alguna-. A ello se une el hecho, antes expuesto, de la dudosa existencia de esos "otros» fondos que son posibles de crear por las CNA, y que hubieran podido afectarse para fines educativos.

Luego, no es de extrañar que desde el inicio del proceso de constitución de las CNA se haya preterido - al menos en esta provincia - la formación para la vida en cooperativa de los socios, y que las acciones de capacitación estén pensadas desde fuera, con las miras puestas en la superación de los directivos de las mismas ${ }^{34}$.

Como correlato de las carencias apuntadas, las socias de las CNA adolecen de una falta de preparación general para su desempeño como cooperativistas — similar al de sus colegas hombre-, con el añadido de que sufren las consecuencias de que ninguno de los/las implicados(as) reciba - tampoco - una formación para hacer frente al desarrollo cooperativo con un adecuado enfoque de género.

\section{A modo de conclusiones}

Como síntesis de lo expuesto es posible sostener que:

- Los marcos normativos generales que regulan a la CNA no tiene una proyección particular dirigida a dar tratamiento a las cuestiones de género. Dicho lo anterior, ha de admitirse -igualmente- que dejan amplios espacios a la autorregulación en los puntos neurálgicos que marcan el desenvolvimiento de los hombres y las mujeres asociados sobre la base de principios de igualdad y participación.

- La relación entre enfoque de género y las Cooperativas No Agropecuarias en Pinar del Río están en sus inicios, toda vez que el primero de estos elementos aun no es conscientemente asumido como un factor a considerar, potenciar y perfeccionar como parte del quehacer cooperativo. No obstante, se advierte que casi todos los aspectos analizados presentan limitaciones en su desarrollo hasta el momento, si se considera todo lo que es posible alcanzar.

- Las mayores fortalezas en este sentido se observan asociadas a la dinámica social que ha establecido conquistas en materia de igualdad que, como ya asumidas, se reproducen en las CNA.

34 Rodríguez Musa, O. y Rivera Gort, J.J.: ob. cit., p. 210. 
Queda entonces bajo la responsabilidad del movimiento cooperativista ampliar estos horizontes aprovechando todas las facilidades de que dispone en este sentido.

- Para la consecución de lo apuntado, en cuanto a materializar el enfoque de género en otras importantes aristas de la referida relación, la educación cooperativa se hace esencial, de ahí la urgencia de incentivarla.

\section{Bibliografía}

Alianza Cooperativa Internacional. Declaración Sobre la Identidad Cooperativa, Manchester, 1995, recuperado de http://www.elhogarobrero1905.org.ar, en fecha 19 de diciembre de 2009.

Alianza Cooperativa Internacional para las Américas. Ley Marco para las cooperativas de América Latina. 1. ${ }^{a}$ ed., Alianza Cooperativa Internacional para las Américas. San José, 2009.

APELQVIST, K.: «Estrategias para una autoridad compartida entre hombres y mujeres en las cooperativas». Revista de Cooperación Internacional, n. ${ }^{\circ} 1$, 1996, pp. 35-47.

Arroyo Sánchez, P.: "Perfil de la situación de la mujer en las cooperativas de trabajo en España», Revista de Estudios Cooperativos (REVESCO), n. ${ }^{\circ} 105$, 2011, pp. 115-142.

Bastidas Delgado, O.: La Especificidad Cooperativa Apuntes para un Cooperativismo Alternativo, Centro de Estudios de la Participación, la Autogestión y el Cooperativismo (CEPAC), Universidad Central de Venezuela, 2009.

COCETA: Estudio sobre la realidad social y laboral de las mujeres en el cooperativismo de trabajo asociado en España, Madrid, 2004.

CotUla, L.: Género y legislación. Los derechos de la mujer en la agricultura. FAO, Roma, 2007.

Elio Cemboraln, E.: Perspectiva de género en el cooperativismo: retos y controversias, recuperado de http://www.euskonews.com/0641zbk/gaia64101es. html, en fecha 19 de junio de 2018.

Elo Cemborain, E.: «Responsabilidad social en las cooperativas: igualdad de oportunidades entre hombres y mujeres», GEZKI, n. ${ }^{\circ} 2$, 2006, pp. 35-71.

Esteban Salvador, M.L.: "¿Son las cooperativas más favorables a la presencia de mujeres en los consejos que otras entidades?». Revista de Estudios Cooperativos (REVESCO), n. ${ }^{\circ} 110,2013$, pp. 96-128.

Esteban Salvador, M.L., Gargallo Castel, A. y Pérez Sanz, F.J.: «¿Presentan las cooperativas contextos favorables para la igualdad de género?: Especial referencia a la provincia de Teruel»». CIRIEC-España, Revista de Economía Pública, Social y Cooperativa, n. ${ }^{\circ} 88,2016$, pp. 61-92.

García Müller, A.: «¿Cómo determinar la identidad de una cooperativa?». Il Taller Internacional de Derecho Cooperativo, Pinar del Río, 7, 8 y 9 de noviembre de 2018. 
Gómez Álvarez, J. y SÁnchez Barrios, M.: «La participación de la mujer en los consejos de administración de empresas del lbex-35». Revista de Humanidades, n. 16, 2009, pp. 1130-5029.

Hernández Aguilar, O.: "La relación género y cooperativa en el ordenamiento jurídico cubano. Una aproximación a su realidad y a sus perspectivas ante el proceso de perfeccionamiento del modelo económico en el país». Boletín de la Asociación Internacional de Derecho Cooperativo, N. ${ }^{\circ} 46,2012$, pp. 297-320.

Hernández Aguilar, O. y Valle Ríos, D.: «La Cooperativa No Agropecuaria Atelier «La Moda». Aproximación a la experiencia de una cooperativa de mujeres». Apuntes de Derecho Cooperativo para Cuba, Ediciones Loynaz, Pinar del Río, 2018, pp. 264-288.

KRISHNAN, H. y PARK, D.: "A few good women -on top management teams». Journal of Business Research, n. ${ }^{\circ}$ 58, 2005, pp. 1712-1720.

Lassaletta García, P.J.: El acceso a la condición de socio en la sociedad cooperativa de trabajo asociado. Junta de Andalucía, Madrid, 2010.

Mateos de Cabo, R., IturRioz del CAMpo, J. y Gimeno Nogués, R.: «La participación financiera y el papel de la mujer en la toma de decisiones de las sociedades cooperativas: los consejos de administración». Revista Europea de Dirección y Economía de la Empresa, vol. 18, núm. 3, 2009, pp. 65-82.

Mesa TejedA, N.T.: «Reflexiones críticas en torno a la regulación de las cooperativas no agropecuarias en Cuba». Boletín de la Asociación Internacional de Derecho Cooperativo, n. ${ }^{\circ} 48,2014$, pp. 227-243

Murillo Jorge, M.: «Intervención del miembro del Buró Político del Comité Central del Partido Comunista de Cuba y Jefe de la Comisión de Implementación y Desarrollo, en el X Periodo Ordinario de Sesiones de la VIII Legislatura de la Asamblea Nacional del Poder Popular, en el Palacio de Convenciones». Periódico Granma. 21 de diciembre de 2017. Recuperado de http://www.granma.cu/cuba/2017-12-22/los-problemas-quehemos-enfrentado-en-la-actualizacion-son-mas-complejos-y-masprofundos-que-loque-habiamos-pensado-22-12-2017-02-12-01, el 22 de enero de 2018.

PiñeIRo HaRneCKer, C.: Diagnóstico preliminar de las cooperativas no agropecuarias en La Habana, Cuba. Recuperado de https://www.ceec.uh.cu/file/569/ download?token=VfqEKMZE, el 18 de abril de 2016.

RIBAS, M.: "Mujeres y cooperativismo en la Comunidad Autónoma del País Vasco». Revista Vasca de Economía Social, n. ${ }^{\circ}$ 2, 2006, pp.153-175.

Rodríguez MusA, O.: «Socialismo, Cooperativismo y Derecho. Dialéctica necesaria para la actualización del modelo económico cubano». Boletín de la Asociación Internacional de Derecho Cooperativo, n. ${ }^{\circ} 46,2012$, pp. 79-105.

Rodríguez Musa, O. y Hernández Aguilar, O.: «Apuntes críticos sobre la implementación jurídica del principio cooperativo de «adhesión voluntaria y abierta» en Cuba». Boletín de la Asociación Internacional de Derecho Cooperativo, No. 51, 2017, pp. 79-98.

Rodríguez MusA, O. y Rivera GORT, J.J. (2015): "Historia y realidad jurídica de la cooperativa en Cuba. Aproximación desde la experiencia práctica a las no- 
veles cooperativas no agropecuarias en Pinar del Río». Boletín de la Asociación Internacional de Derecho Cooperativo, n. ${ }^{\circ} 49$, pp. 195-222.

SILVA González, J.L.: Fundamentos para una crítica propositiva al enfoque de género en el ordenamiento jurídico cubano: Estrategia para su implementación. Tesis presentada en opción al título de Licenciado en Derecho. Universidad de Pinar del Río, 2014.

Velázquez Quintián, S. y BarRera Rodríguez, S.: «Una mirada con enfoque de género a la legislación y la realidad de una cooperativa no agropecuaria cubana». Revista Idelcoop, n. ${ }^{\circ} 218,2016$, pp. 79-92.

VuotTo, M.: "Las cooperativas no agropecuarias y la transformación económica en cuba: políticas, procesos y estrategias». Revista de Estudios CooperatiVOS (REVESCO), recuperado de http://dx.doi.org/10.5209/rev_REVE.2016. v120.49697, el 18 de abril de 2016.

YÁNEZZ, S. "Consideraciones sobre flexibilidad laboral planteadas desde una mirada de género». En ENSIGNIA, J. y YÁÑEZ, S.: Sindicalismo, género y flexibilización en el Mercosur y Chile. Inserción laboral femenina. Fundación Friedrich Ebert (Representación Chile) y Centro de Estudios de la Mujer (CEM), Santiago de Chile, 1999, pp. 97-110.

\section{Legislación}

- Constitución de la República de Cuba (vigente), Gaceta Oficial de la República de Cuba, Edición Especial, La Habana, 24/02/1976, reformada por la Ley de Reforma Constitucional, Gaceta Oficial de la República de Cuba, Edición Extraordinaria, n. ${ }^{\circ}$ 9, La Habana, 13/07/1992 y por la Ley de Reforma Constitucional, Gaceta Oficial de la República de Cuba, Edición Extraordinaria, n. ${ }^{\circ} 10$, La Habana, 16/07/2002.

- Ley No. 116. Código de Trabajo. Gaceta Oficial No. 29 Extraordinaria de 17 de junio de 2014.

- Decreto-Ley No. 305. De las Cooperativas No Agropecuarias. Gaceta Oficial No. 53 Extraordinaria de 11 de diciembre de 2012.

- Decreto No. 309. Reglamento de las Cooperativas No Agropecuarias. Gaceta Oficial No. 53 Extraordinaria de 11 de diciembre de 2012.

- Indicación Metodológica de la Dirección de los Registros de la Propiedad, Mercantil y Patrimonio. Ministerio de Justicia de 10 de junio de 2013.

- Indicación Metodológica n. ${ }^{\circ}$ 4. Dirección de Notarias del MINJUS de 3 de septiembre de 2015. 